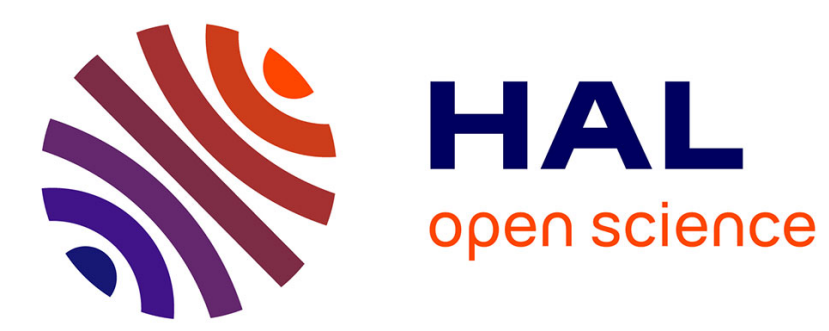

\title{
Editorial: Relire les décolonisations d'Afrique francophone au prisme du genre
}

Emmanuelle Bouilly, Ophélie Rillon

\section{To cite this version:}

Emmanuelle Bouilly, Ophélie Rillon. Editorial: Relire les décolonisations d'Afrique francophone au prisme du genre. Le Mouvement social, 2016, 255 (2), pp.3-16. 10.3917/lms.255.0003 . halshs02386745

\section{HAL Id: halshs-02386745 \\ https://shs.hal.science/halshs-02386745}

Submitted on 29 Nov 2019

HAL is a multi-disciplinary open access archive for the deposit and dissemination of scientific research documents, whether they are published or not. The documents may come from teaching and research institutions in France or abroad, or from public or private research centers.
L'archive ouverte pluridisciplinaire HAL, est destinée au dépôt et à la diffusion de documents scientifiques de niveau recherche, publiés ou non, émanant des établissements d'enseignement et de recherche français ou étrangers, des laboratoires publics ou privés. 


\section{Editorial : Relire les décolonisations d'Afrique francophone au prisme du genre}

\section{Emmanuelle Bouilly}

Doctorante à l'Université Paris 1 Panthéon-Sorbonne, Centre européen de sociologie et de science politique (CESSP).

\section{Ophélie Rillon}

Chargée de recherche, laboratoire Les Afrique dans le monde (LAM)-CNRS.

L'étude des luttes sociales et politiques en Afrique a connu un regain d'intérêt ces dernières années ${ }^{1}$. Ce dynamisme tient autant à l'ampleur des mouvements sociaux qui se sont déroulés récemment sur le continent - dont les «printemps arabes ${ }^{2}$ » ont constitué la face la plus visible - qu'à un renouvellement des terrains et des objets de la sociologie de l'action collective, jusqu'alors davantage centrée sur les pays du Nord. Les travaux actuels sur l'Afrique témoignent cependant d'un intérêt inégal ${ }^{3}$ pour les mobilisations de femmes et/ou le genre comme «catégorie utile d'analyse ${ }^{4}$ » alors même que les études sur le sexe du militantisme se sont multipliées durant la décennie $1990^{5}$. C'est aux historiennes et

1 M. Bennani-Chraïbi et O. Fillieule (dir.), Résistances et protestations dans les sociétés musulmanes, Paris, Presses de Sciences Po, 2002 ; S. Ellis et S. Van Kessel (dir.), Movers and Shakers : Social Movements in Africa, Leyde-Boston, Brill, 2010 ; R. Banégas, M.-E. Pommerolle et J. Siméant (dir.), « Lutter dans les Afriques », Genèses, n 81, 2010 ; M. Larmer (dir.), « Africa’s Struggles », The Review of African Political Economy, vol. 37, $\mathrm{n}^{\circ}$ 125, 2010 ; B. Rubbers et A. Roy (dir.), «Les chantiers du syndicalisme en Afrique », Revue Tiers Monde, $\mathrm{n}^{\circ} 224,2015$.

2 Pour n'en citer que deux en français : M. Bennani-Chraïbi et O. Fillieule (dir.), «Retour sur les situations révolutionnaires arabes », Revue française de science politique, vol. 62, $\mathrm{n}^{\circ}$ 5-6, 2012 ; A. Allal et T. Pierret (dir.), Au cœur des révoltes arabes : devenir révolutionnaires, Paris, Armand Colin, 2013.

3 Parmi les travaux faisant discuter théories des mouvements sociaux et du genre, on peut relever K. Fallon, Democracy and the Rise of Women's Movements in Sub-Saharan Africa, Baltimore, Johns Hopkins University Press, 2008 ; F. C. Steady, Women and Collective Action in Africa, New York, Palgrave Macmillan, 2006.

4 J. Scott, «Le genre : une catégorie utile d'analyse historique », Les Cahiers $d u$ GRIF, vol. 37, n 37-38, 1988, p. 125-153.

5 Ces travaux, faisant dialoguer les outils de la sociologie et ceux des études de genre, s'inspirent des recherches initiées par Danièle Kergoat sur les 
Editorial : Relire les décolonisations d'Afrique francophone au prisme du genre

historiens qui ne recourent pas au « kit conceptuel » des théories des mouvements sociaux, que l'on doit les premières études sur les mobilisations ou l'engagement militant de femmes africaines. L'accent était alors mis sur l'activité politique des colonisées, ainsi que sur leur rôle dans les résistances à la colonisation et les luttes d'indépendance. Depuis quelque temps cependant, les travaux historiques centrés sur le politique semblent s'essouffler au profit d'une histoire des femmes et du genre plus attentive aux dynamiques sociales et culturelles $^{6}$. Le renouvellement de l'histoire coloniale et impériale s'est en quelque sorte accompagné d'un certain abandon de l'étude des mouvements sociaux et de l'action protestataire, relégués au rang des thématiques obsolètes ${ }^{7}$. Deux recherches inédites sont néanmoins venues avec bonheur ouvrir de nouvelles pistes pour écrire l'histoire des luttes sociales et politiques en Afrique, sans pour autant intégrer à la réflexion une perspective de genre ${ }^{8}$.

Les contributions réunies dans ce numéro ${ }^{9}$ souhaitent, à rebours de ces multiples décalages, promouvoir un dialogue interdisciplinaire et un décloisonnement des différentes bibliothèques - études africaines, études de genre, histoire (coloniale, impériale ou sociale du politique), sociologie de l'action collective ${ }^{10}$. En prenant pour point d'entrée commun

«mouvements sociaux sexués ». Si les historiennes des femmes (à l'instar de Michelle Perrot) ont de longue date étudié les formes d'engagement féminin (féministes ou non) ainsi que le rôle joué par les femmes dans les mouvements sociaux, c'est à partir des années 2000 que l'outil du genre a véritablement fait son entrée dans la discipline historique. Les travaux de Michelle ZancariniFournel sont une belle illustration de la manière dont, en histoire, peuvent s'articuler les études de genre et les recherches sur le militantisme ( Genre et politique : les années 1968 », Vingtième Siècle. Revue d'histoire, vol. 3, n 75, 2002, p. 133-143) ; D. Kergoat,

F. Imbert, H. Le Doare et D. Senotier, Les infirmières et leur coordination. 1988-1989, Paris, Lamarre, 1992 ; X. Dunezat, «Des mouvements sociaux sexués », Recherches féministes, vol. 11, n² 2, 1998 ; O. Fillieule et P. Roux (dir.), Le sexe du militantisme, Paris, Presses de Sciences Po, 2009. La bibliographie anglophone est particulièrement riche sur le sujet. Voir par exemple R. L. Einwohner, J. A. Hollander et T. Olson, «Engendering Social Movements: Cultural Images and Movement Dynamics », Gender and Society, vol. 14, n 5, 2000, p. 679-699.

6 P. Barthélémy, L. Capdevila et M. Zancarini-Fournel (dir.), «Femmes, genre et colonisations », Clio. Histoire, Femmes et Sociétés, n 33, 2011, p. 7-22.

7 En témoigne par exemple la trajectoire scientifique d'un historien comme Frederick Cooper, passé de l'étude des mouvements sociaux au Kenya aux politiques coloniales d'encadrement des travailleurs d'Afrique de l'ouest (et notamment les grèves de cheminots) puis, récemment, à l'examen des débats politiques autour des enjeux liés à la citoyenneté dans le processus de décolonisation.

8 F. Blum, Révolutions africaines. Congo, Sénégal, Madagascar, années 1960-1970, Rennes, Presses universitaires de Rennes, 2014 (voir sa recension dans ce numéro); K. van Walraven, The Yearning for Relief : A History of the Sawaba Movement in Niger, Leyde-Boston, Brill, 2013.

9 Nous remercions vivement Pascale Barthélémy pour sa contribution à la réalisation de ce dossier.

10 Cette réflexion a été menée durant deux ans au cours d'un séminaire réunissant «jeunes » chercheurs et chercheuses en sciences sociales qui s'étaient donné pour objectif d'interroger le sexe des mobilisations 
l'engagement de femmes dans les colonies françaises, les auteurs de ce numéro entendent relire les décolonisations au prisme du genre ${ }^{11}$. Il ne faudrait pas y voir la volonté de féminiser l'histoire des décolonisations en y ajoutant des actrices oubliées, et encore moins celle de «colorer » ou d' « exotiser » une sociologie du genre ou de l'action collective en voie de routinisation. Comme le souligne Dominique Godineau à propos de la Révolution française : «Il ne suffit pas de dire que les femmes y ont ou n’y ont pas participé. Il ne suffit pas non plus de mettre en évidence le poids du facteur masculin-féminin. Il faut questionner l'histoire pour tenter de dégager l'articulation entre les rapports des sexes et l'événement ${ }^{12} »$. Avec ce dossier, il s'agit de saisir combien les processus de décolonisation - mobilisations sociopolitiques, guerres, réformes de l'Empire - ont été en même temps des moments de réaffirmation, de recomposition et de vacillement des rapports de genre. Dès lors, l'enjeu de ce numéro est double. Il s'agit d'une part de comprendre la dialectique des rapports sociaux de sexe en insistant sur la manière dont les assignations de genre structurent les mobilisations, qu'elles soient exclusivement féminines ou mixtes. D'autre part, ce numéro montre que le genre, conçu comme un rapport social de pouvoir fondé sur la différence perçue entre les sexes, est produit dans la période singulière de renégociation des relations entre métropole et colonies. En optant pour une temporalité large qui s'étend des années 1940 aux années 1970, il ne s'agit ni de résumer les décolonisations à «l'événement indépendance », trop souvent pensé comme une rupture, ni de circonscrire les mobilisations de cette période aux seules revendications d'autonomie ou de libération nationale. Ce dossier entend porter un regard ouvert sur le bouillonnement sociopolitique de ces décennies pour rappeler à quel point les processus de décolonisation constituèrent des moments d'ouverture et représentent des terrains d'investigation privilégiés pour examiner comment se renégocient les rapports de genre.

collectives en Afrique. Organisé à l'Université Paris 1 entre septembre 2011 et juin 2013, ce séminaire a reçu le concours logistique et financier de l'IMAF, du CRPS et du Collège des écoles doctorales. Il y associait également comme organisatrice Marie Brossier, que nous remercions. D'autres contributions, portant sur une période plus récente, seront publiées fin 2016 dans la Review of African Political Economy.

11 Ce dossier, se conformant aux choix éditoriaux du Mouvement social, n'adopte pas « l'écriture épicène ». Rappelons que celle-ci permet, à nos yeux, de donner une visibilité égale aux femmes et aux hommes dans l'analyse en soulignant que le langage n'est pas neutre mais révélateur des rapports de genre.

12 D. Godineau, «Filles de la liberté et citoyennes révolutionnaires », in G. Fraisse et M. Perrot (dir.), Histoire des femmes en Occident. Le xix siècle, Paris, Plon, 1991, p. 27. 


\section{Femmes, genre et décolonisations : déplacer la focale}

«Plus encore peut-être que la période coloniale stricto sensu, le genre a été un outil peu utilisé pour comprendre les processus de décolonisation ${ }^{13}$. » Si ce constat, partagé par plusieurs historiennes et historiens, demeure d'actualité, il concerne surtout la recherche en langue française. Les ouvrages de synthèse publiés en France ces dernières années sur les décolonisations en sont le triste reflet. À quelques exceptions près, les femmes n'y occupent aucune place, et la fabrique des identités sexuées (féminines ou masculines) dans les processus d'indépendance n'est quasiment pas interrogée ${ }^{14}$. À l'inverse, depuis les années 1970, les travaux sur les femmes ou le genre dans les luttes anticoloniales et pour les libérations nationales se sont multipliés outre-Atlantique. Alors que Susan Geiger, l'une des pionnières sur ces thématiques, publiait une synthèse historiographique sur ces questions dès $1990^{15}$, l'histoire des femmes en Afrique commençait à peine à émerger en France $^{16}$. Une historiographie particulièrement riche existe désormais sur la rébellion Mau Mau dans le Kenya des années $1950^{17}$, les mouvements nationalistes en Afrique

13 A. Lauro, «Introduction. Colonialismes », Sextant, 2008, $\mathrm{n}^{\circ} 25$, p. 9. Voir aussi Joly, « Femmes et décolonisation en Afrique occidentale française : autour de la marche des femmes de Grand-Bassam (décembre 1949) », in M. Bergère et L. Capdevila (dir.), Genre et événement. Du masculin et du féminin en histoire des crises et des conflits, Rennes, Presses universitaires de Rennes, 2006, p. 105-117 ; P. Levine, «Gendering Decolonisation», Histoire@Politique. Politique, culture, société, n 11, 2010, www.histoirepolitique.fr.

14 Seuls deux ouvrages contiennent chacun une contribution sur les femmes: B. Stora, «Mémoires comparées : femmes françaises, femmes algériennes. Les écrits de femmes, la guerre d'Algérie et l'exil », in C.-R. Ageron et M. Michel (dir.), L'ère des décolonisations, Paris, Karthala, 1995, p. 172-194 ; K. Langewiesche, «Actrices du quotidien. Congrégations féminines en Haute-Volta lors de l'indépendance », in O. Goerg, J.-L. Martineau et D. Nativel (dir.), Les indépendances en Afrique. L'événement et ses mémoires 1957/1960-2010, Rennes, Presses universitaires de Rennes, 2013, p. 117-134.

15 S. Geiger, «Women and African Nationalism », Journal of Women's History, vol. 2, n 1, 1990, p. $227-$ 244.

16 Y. Knibiehler et R. Goutalier, La femme au temps des colonies, Paris, Stock, 1985 ; C. CoqueryVidrovitch, Les Africaines. Histoire des femmes d'Afrique noire du XIX $X^{e}$ au XX siècle, Paris, Desjonquères, 1994.

17 K. Santilli, « Kikuyu Women in the Mau Mau Revolt: A Closer Look », Ufahamu, vol. 8, n 1, 1977-1978, p. 143-174; T. Kanogo, « Kikuyu Women and the Politics of Protest: Mau Mau », in S. MacDonald, P. Holden et S. Ardener (dir.), Images of Women in Peace and War: Cross-cultural and Historical Perspectives, Basingstoke, Macmillan Education, 1987, p. 78-99; L. White, «Separating the Men from the Boys: Constructions of Gender, Sexuality, and Terrorism in Central Kenya, 1939-1959 », International Journal of African Historical Studies, vol. 23, n 1, 1990, p. 1-25; C. A. Presley, Kikuyu Women, the Mau Mau Rebellion, and Social Change in Kenya, San Francisco, Westview Press, 1992; W. W. Otieno, Mau Mau's Daughter. A Life History, Boulder, Lynne Rienner, 1998. 
Editorial : Relire les décolonisations d'Afrique francophone au prisme du genre

anglophone ${ }^{18}$ (Tanzanie et Zimbabwe notamment) ou lusophone ${ }^{19}$, ou encore la lutte contre l'apartheid en Afrique du sud ${ }^{20}$. Dans ce champ où dominent les recherches sur l'Afrique non francophone et les luttes (armées ou non) de libération nationale, les travaux sur la guerre d'Algérie font figure d'exception pour avoir intégré à la réflexion, même tardivement, les femmes et le genre ${ }^{21}$.

C'est encore aux historiennes américaines que l'on doit d'avoir défriché le terrain des mobilisations féminines dans l'Afrique colonisée par les Français ${ }^{22}$. Leur démarche, circonscrite aux pays de l'ancienne Afrique occidentale française (AOF), mérite d'être poursuivie et étendue à d'autres territoires. En effet, les décolonisations d'Afrique francophone ont pris des formes diverses, plus ou moins violentes ou négociées, que ce dossier vise à embrasser. Des décolonisations prétendument « pacifiques » de l'AOF (P.

18 S. Geiger, «Anti-Colonial Protest in Africa: A Female Strategy Reconsidered », Heresies, n ${ }^{\circ}$, 1980, p. 22-25; id., «Women in Nationalist Struggle: TANU Activists in Dar es Salaam », International Journal of African Historical Studies, vol. 20, n 1, 1987, p. 1-26; E. Batezat et al., "Women and Independence: The Heritage and the Struggle », in C. Stoneman (dir.), Zimbabwe's Prospects: Issues of Race, Class, State, and Capital in Southern Africa, Londres, Macmillan, 1988, p. 153-173; K. Bond-Stewart, Independence is not only for One Sex, Harare, Zimbabwe Publishing House, 1987; T. Lyons, Guns and Guerilla Girls: Women in the Zimbabwean National Liberation Struggle, New Jersey, Africa World Press, 2004; S. Ranchod-Nilson, «This Too Is a Way of Fighting: Rural Women's Participation in Zimbabwe's Liberation War », in M. A. Tétreault (dir.), Women and Revolution in Africa, Asia, and the New World, Columbia, University of South Carolina Press, 1994, p. 62-88; T. Scarnecchia, «Poor Women and Nationalist Political Movement in Salisbury, Rhodesia 1950-1956 », Journal of African History, vol. 37, n² 2, 1996, p. 283-310.

19 M. J. Arthur, « Mozambique: Women in the Armed Struggle », in P. McFadden (dir.), Gender in Southern Africa: A Gendered Perspective, Harare, Sapes Books, 1998, p. 67-82; K. Sheldon, «Women and Revolution in Mozambique: A Luta Continua », in M. A. Tétreault (dir.), Women and Revolution ..., op. cit., p. 33-61; S. Urdang, «The Last Transition? Women and Development in Mozambique », Review of African Political Economy, $\mathrm{n}^{\circ}$ 27-28, 1983, p. 8-32; A. Ly, « Promise and betrayal: Women fighters and national liberation in Guinea Bissau », Feminist Africa, n ${ }^{\circ}$ 19, 2014, p. 24-42; S. Urdang, Fighting Two Colonialisms: Women in Guinea-Bissau, New York-Londres, Monthly Review, 1979.

20 J. C. Wells, «Why Women Rebel: A Comparative Study of South African Women's Resistance in Bloemfontein (1913) and Johannesburg (1958) », Journal of Southern African Studies, vol. 10, 1982, p. 3955; S. Hassim, « Nationalism, Feminism and Autonomy: The ANC in Exile and the Question of Women », Journal of Southern African Studies, vol. 30, n 3, p. 433-455.

21 D. Amrane, Les Femmes algériennes dans la guerre, Paris, Plon, 1991 ; R. Branche, «La Masculinité à l'épreuve de la guerre sans nom », Clio. Histoire, Femmes et Sociétés, n 20, 2004, p. 111-122; N. Vince, Our Fighting Sisters. Nation, Memory and Gender in Algeria, 1954-2012, Manchester, Manchester University Press, 2015.

22 M. Dobert, «Civic and Political Participation of Women in French-Speaking West Africa », thèse de doctorat, George Washington University, 1970; E. Schmidt, Mobilizing the Masses: Gender, Ethnicity and Class in the Nationalist Movement in Guinea, 1939-1958, Portsmouth, Heinemann, 2005; B. Cooper, «The Politics of Difference and Women's Associations in Niger: Of 'Prostitutes', the Public, and Politics », Signs, vol. 20, n 4, 1995, p. 851-882. Ainsi que l'article précurseur en France d'O. Goerg, «Femmes africaines et politique : les colonisées au féminin en Afrique occidentale », Clio. Histoire, Femmes et Sociétés, n 6, 1997, p. 105-125. 
Barthélémy, O. Rillon), à l'indépendance refusée dans le cas de Mayotte (M. Idriss), en passant par les guerres de décolonisation en Algérie (P.-J. Le Foll-Luciani, N. Sidi Moussa) et au Cameroun ${ }^{23}$ (R. Ndengue, C. Walker-Said), les articles de ce dossier offrent une «narration historique multiple et contradictoire » des processus de décolonisation ${ }^{24}$. Alors que l'historiographie avait jusqu'à présent mis l'accent sur l'inclusion des femmes dans des organisations nationalistes et leur participation aux révoltes ou rébellions violentes, ce dossier propose un changement de perspective en orientant le regard sur des formes de mobilisations plus «ordinaires » et «quotidiennes ». En examinant les décolonisations en dehors des sites de conflits les plus évidents, l'objectif est de saisir « l'histoire par en bas ${ }^{25}$ » des expériences et des luttes féminines.

Les cas d'étude réunis ici ont pour contexte les réformes introduites dans l'Empire français au lendemain de la Seconde Guerre mondiale qui ont favorisé le développement d'activités militantes pour les hommes et les femmes des colonies. Selon les territoires, le régime de l'indigénat fut progressivement démantelé à partir de 1944, date à laquelle un décret leva aussi l'autorisation maritale pour les femmes souhaitant se syndiquer, à la condition néanmoins qu'elles soient lettrées en français. Le droit de réunion et d'organisation était étendu aux colonies en 1946. La même année, grâce à la loi Lamine Guèye, l'ensemble des populations colonisées accédait à la citoyenneté, sans que ce statut ne leur confère le droit de vote. Ces transformations législatives désormais bien connues ne sauraient être les seuls facteurs explicatifs du bouillonnement des mobilisations de cette période. Elles ont néanmoins ouvert le champ des possibles - de façon toutefois inégale pour les hommes et les femmes. Les débats et les mobilisations qui entourent la question du droit de vote des femmes dans les colonies sont ainsi révélateurs des rapports de genre et de « race $^{26} »$ qui se jouent dans le contexte de réforme des empires (R. Ndengue ${ }^{27}$. Si l'histoire algérienne est singulière dans le paysage colonial, du fait de son statut de département ayant autorisé

23 T. Deltombe, M. Domergue et J. Tatsitsa, Kamerun! Une guerre cachée aux origines de la Françafrique (1948-1971), Paris, La Découverte, 2011.

24 S. Awenengo, P. Barthélémy et C. Tshimanga (dir.), Écrire l'histoire de l'Afrique autrement?, Paris, L'Harmattan, 2004, p. 12.

25 E. P. Thompson, « History from Below », Times Literary Supplement, 7 avril 1966, p. 279-280.

26 Nous employons le terme de « race » et celui de « genre » comme des constructions sociales et historiques. 27 J. Lacroix et S. Mbaye, «Le vote des femmes au Sénégal », Éthiopiques, n 6, 1976, p. 26-43 ; F. Cooper, Citizenship between Empire and Nation: Remaking France and French Africa, 1945-1960, Princeton, Princeton University Press, 2014, p. 59-63. 
une vie politique et électorale ancienne, les transformations législatives opérées aprèsguerre y ont aussi profondément renouvelé les possibilités d'engagement des populations locales.

\section{Déconstruire les grands récits nationalistes}

Alors que l'historiographie a jusqu'ici mis l'accent sur la «volonté de rupture et la force des nationalismes ${ }^{28} »$, plusieurs travaux ont récemment souligné combien l'histoire des décolonisations était infiniment plus complexe. C'est notamment l'apport du travail de Frederick Cooper dans Citizenship between Empire and Nation. Ce livre montre que, dans la redéfinition des relations entre la métropole française et ses colonies, la revendication de l'indépendance n'a été que tardivement formulée par les élites ouest-africaines. La critique du système colonial n'induisait pas mécaniquement la revendication d'un État-nation indépendant. L'auteur met l'accent sur l'extraordinaire capacité d'invention politique des dirigeants africains dans les décennies qui suivirent la Seconde Guerre mondiale. Le cas de la lutte pour la départementalisation de Mayotte et le mouvement des «chatouilleuses» étudié dans ce numéro par Mamaye Idriss rappellent combien d'autres présents étaient possibles. À bien des égards, les articles de ce dossier se situent dans le prolongement de la réflexion initiée par $\mathrm{F}$. Cooper, tout en déplaçant le regard hors de la scène parlementaire pour réinvestir la rue et les mobilisations sociopolitiques. En procédant à une histoire sociale centrée sur les conflits locaux et les expériences de militantes et militants africains, l'ensemble de ces articles entend se défaire des grands récits nationalistes produits par les leaders de l'indépendance ${ }^{29}$.

En effet, au moins jusqu'aux années 1980, l'écriture de l'histoire de ces luttes a constitué une puissante matrice de fabrique de héros masculins et de promotion de la vision des vainqueurs, anciens colonisés ${ }^{30}$. Ce processus a notamment été critiqué par les tenants des

28 P. Barthélémy, « Autour d'un livre », Politique africaine, vol. 4, n 136, 2014, p. 179.

29 Sur la question du genre et de la nation dans une perspective historique et sociologique, voir L. Auslander et M. Zancarini-Fournel (dir.) « Le genre de la nation », Clio. Histoire, Femmes et Sociétés, n 12, 2000, p. 513 ; A. Le Renard et E. Marteu (dir.), « Genre et nation : approches sociologiques », Sociétés contemporaines, $\mathrm{n}^{\circ} 94,2014$, p. 5-18.

30 F. Cooper, «Conflict and Connection: Rethinking Colonial African History », The American Historical Review, vol. 99, $\mathrm{n}^{\circ}$ 5, 1994, p. 1516-1545; J. Allman, « Between the present and history: African Nationalism and Decolonization », in J. Parker et R. Reid (dir.), The Oxford Handbook of Modern African History, Oxford, Oxford University Press, 2013, p. 224-240. 
subaltern studies à l'instar de Partha Chatterjee qui souligne combien « the story of nationalist emancipation is necessarily a story of betrayal. Because it could confer freedom only by imposing at the same time a whole new set of controls, it could define a cultural identity for the nation only by excluding many from its fold ${ }^{31} »$. L'histoire des luttes internes aux populations africaines reste encore largement à écrire. La focale est en effet restée longtemps concentrée sur les conflits avec l'administration coloniale, générant l'image réductrice d'un front uni contre la colonisation et participant à homogénéiser les luttes des populations colonisées ${ }^{32}$. Si les subalternists ont montré de longue date que les sociétés colonisées étaient traversées par d'importantes lignes de fractures et que l'adhésion au projet nationaliste était loin d'être univoque, ils ont largement oublié, avec d'autres, que ces sociétés étaient aussi composées de femmes ${ }^{33}$.

Le renversement méthodologique induit par l'écriture d'une histoire des subalternes conjuguée au féminin est en grande partie rendu possible par la collecte de sources inédites. Les auteurs de ce dossier ont ainsi fait feu de tout bois en alliant la recherche documentaire au sein de fonds privés familiaux, individuels ou d'organisations féminines ou mixtes, à l'exploration des écrits féminins (articles de presse, autobiographies) et à la réalisation d'entretiens biographiques. Ces matériaux révèlent les tensions internes, et parfois intimes, générées par l'engagement féminin et font émerger des expériences militantes singulières. Croisés avec les archives de l'administration coloniale, ils permettent de relire sous un autre jour une documentation qui nous renseigne parfois «malgré elle » sur les mobilisations de femmes africaines ${ }^{34}$. Ces sources plurielles contribuent à multiplier les points d'entrée pour interroger la complexité des imaginaires sociopolitiques de ces décennies.

31 P. Chatterjee, The Nation and its Fragments: Colonial and Postcolonial Histories, Princeton, Princeton University Press, 1993, p. 154.

32 À l'exception des travaux sur l'Algérie auxquels ont contribué deux des auteurs de ce dossier : N. SidiMoussa, «Devenirs messalistes (1925-2013) : sociologie historique d'une aristocratie révolutionnaire», thèse de doctorat de science politique, Université Paris 1, 2013 ; P.-J. Le Foll-Luciani, Les juifs algériens dans la lutte anticoloniale. Trajectoires dissidentes (1934-1965), Rennes, Presses universitaires de Rennes, 2015.

33 La critique a été formulée précocement par G. C. Spivak, «Subaltern Studies: Deconstructing Historiography », Subaltern Studies, vol. 4, 1985, p. 330-363. Voir aussi M. Diouf (dir.), L'historiographie indienne en débat. Colonialisme, nationalisme et sociétés postcoloniales, Paris-Amsterdam, Karthala-Sephis, 1999.

34 M. Rodet, "'C'est le regard qui fait l'histoire'. Comment utiliser des archives coloniales qui nous renseignent malgré elles sur l'histoire des femmes africaines (archives) », Terrains \& travaux, vol. $1, \mathrm{n}^{\circ} 10$, 2006, p. 18-35. 


\section{Sortir du face-à-face entre la métropole et ses colonies}

En analysant les relations entretenues par des Africaines avec la Fédération démocratique internationale des femmes (FDIF), Pascale Barthélémy invite à dépasser la seule confrontation entre métropole et colonies. Cette approche transnationale lui permet d'examiner comment des liens politiques et amicaux se sont tissés entre des femmes françaises, européennes et africaines, et ont servi de support à l'engagement politique de ces dernières. Elle montre à quel point l'élaboration de mots d'ordre internationaux communs (le pacifisme, un féminisme maternaliste et différentialiste) a servi de levier à des mobilisations locales anticoloniales dans des contextes africains marqués par une forte répression (en Côte d'Ivoire notamment). À l'occasion des conférences internationales de femmes, ces militantes africaines se sont faites les porte-parole des mobilisations politiques et sociales qui se jouaient dans les territoires colonisés. Cette recherche vient prolonger les pistes ouvertes par Meredith Terretta dans son travail sur les milliers de pétitions envoyées par les Camerounaises au Conseil de tutelle des Nations Unies dans les années 1950 pour dénoncer la politique de l'administration française ${ }^{35}$. En mettant ainsi l'accent sur les liens noués entre femmes au-delà des appartenances religieuses, raciales ou territoriales, plusieurs articles examinent la circulation d'individus, de modes d'action et d'idées entre la métropole et les colonies mais aussi à l'intérieur du monde arabo-musulman ${ }^{36}$ (N. Sidi Moussa), soulignant par-là combien l'histoire des conflits sociopolitiques fut aussi une histoire de connexions, de réseaux (communistes, féministes, religieux, migratoires) et de circulations, rappelant s'il en était encore besoin l'extraversion ancienne du continent africain $^{37}$.

Une autre manière de sortir de l'opposition binaire entre colonisateurs et colonisés consiste à étudier les tensions internes aux sociétés africaines, elles-mêmes traversées par de profonds clivages sociopolitiques pendant cette séquence historique. Une seconde série d'articles déplace ainsi la focale vers des conflits locaux pour examiner les mobilisations

35 M. Terretta, Petitioning for Our Rights, Fighting for Our Nation. The History of the Democratic Union of Cameroonian Women, 1949-1960, Bamenda, Langaa RPCIG, 2013 (voir sa recension dans ce numéro).

36 L. Dakhli et S. Latte Abdallah (dir.), « Des engagements féminins au MoyenOrient $\left(\mathrm{XX}^{\mathrm{e}}-\mathrm{XXI}^{\mathrm{e}}\right.$ siècles) », Le Mouvement social, $\mathrm{n}^{\circ} 231,2010$.

37 J.-F. Bayart, «L'Afrique dans le monde : une histoire d'extraversion », Critique internationale, vol. 5, n 1, 1999, p. 97-120 ; F. Cooper, L'Afrique dans le monde. Capitalisme, empire, État-nation, Paris, Payot et Rivages, 2015. 
féminines in situ à l'échelle de la rue et du quartier. À la croisée d'une double historiographie - celle portant sur les femmes dans les villes coloniales ${ }^{38}$ et celle concernant la violence politique des femmes ${ }^{39}$, Ophélie Rillon et Mamaye Idriss auscultent avec minutie le rôle joué par les militantes dans la conflictualité urbaine et lèvent le voile sur la violence des affrontements qui ont opposé les colonisés. S'inspirant des enseignements méthodologiques de l'historiographie indienne, les auteures relisent les archives coloniales et les récits rétroactifs «à contre fil » (against the grain) ${ }^{40}$. Cette démarche leur permet d'examiner les différents niveaux de signification de leurs sources et de décrypter des pratiques protestataires féminines qui ne nous parviennent que par bribes.

\section{Des mobilisations genrées aux rapports de genre produits par}

\section{la lutte}

Ce numéro explore la manière dont les rapports de genre se déploient, sont produits et reproduits par et dans les luttes sociales et politiques qui se développent à la fin de l'Empire français. Il s'agit de comprendre comment le militantisme et le colonialisme tardif sont traversés par des rapports de genre, et comment ils fabriquent en retour ces rapports. L'ensemble des contributions s'est donné pour objet initial des femmes engagées militantes encartées dans des organisations partisanes ou syndicales, adhérentes d'associations religieuses, caritatives ou de solidarité, ou bien encore des femmes mobilisées de manière plus informelle et ponctuelle - afin d'étudier de manière relationnelle l'organisation des rapports sociaux de sexe. C'est donc en choisissant «d'analys[er] une seule des deux catégories de $\operatorname{sexe}^{41} »$ que les auteurs entendent saisir les rapports de genre tels qu'ils sont déterminés et produits par et dans les luttes sociopolitiques de la période des décolonisations. On suit ainsi le parcours et l'engagement de militantes algériennes communistes (P.-J. Le Foll-Luciani) ou de messalistes syndiquées (N. Sidi

38 C. D. Gondola, «Unies pour le meilleur et pour le pire. Femmes africaines et villes coloniales : une histoire du métissage », Clio. Histoire, Femmes et Sociétés, n 6, 1997, p. 87-104 ; M. Rodet, « Administration et villes coloniales : refuges pour les femmes ? Kayes, Soudan français, (1900-1920) », Sextant, $n^{\circ} 25,2009$, p. 13-27.

39 C. Cardi et G. Pruvost (dir.), Penser la violence des femmes, Paris, La Découverte, 2012.

40 R. Guha, « The Prose of Counter-Insurgency », Subaltern Studies II, Delhi, Oxford University Press, 1983, p. $45-84$.

41 P. Roux et O. Fillieule, «Avant-propos », in Id. (dir.), Le sexe du militantisme, op. cit., p. 17. 
Moussa), d'élites ouest-africaines internationalisées (P. Barthélémy), de militantes politiques instruites (R. Ndengue, O. Rillon, C. Walker-Said) comme issues des classes populaires (M. Idriss, O. Rillon, N. Sidi Moussa), de Camerounaises investies dans le militantisme chrétien et/ou social (R. Ndengue, C. Walker-Said). Il s'agit souvent de minorités politiques et sociologiques (femmes éduquées, opposantes messalistes ou communistes, ouvrières, syndicalistes), sans qu'ait toutefois été abandonné le projet de déceler dans les archives et les mémoires d'autres profils de femmes mobilisées (commerçantes, paysannes, classes populaires urbaines). Ces différents cas d'étude font ressortir un certain nombre d'enseignements qui s'inscrivent dans le prolongement des travaux sur le sexe du militantisme.

Les auteurs montrent combien les mobilisations qu'ils étudient - mobilisations étant ici employées dans un sens inclusif ne se réduisant pas à la forme «mouvement social » telle qu'observée dans les espaces occidentaux ${ }^{42}$ - sont genrées car les contextes sociopolitiques, juridiques ou organisationnels militants dans lesquels elles se produisent, comme les identités, pratiques et relations militantes qui s'y déploient, sont profondément structurés par les rapports sociaux de sexe que ces mobilisations contestent ou consolident. Idriss Mamaye rappelle que le mouvement mahorais des «chatouilleuses » est né des bouleversements à la fois de l'organisation familiale et conjugale et de l'économie domestique et locale engendrés par la décision administrative de transférer le chef-lieu de Mayotte vers une autre île des Comores. C'est alors sur la base d'intérêts économiques et affectifs communs liés à leur position sociale d'épouses ou de concubines que des femmes se sont mobilisées contre ce transfert avant de rejoindre le mouvement politique réclamant la départementalisation de Mayotte. On voit bien ici comment l'économie des rapports de genre structure différemment, selon le sexe et le statut matrimonial, le passage à l'action militante. Pascale Barthélémy montre dans le même sens combien les participantes françaises et ouest-africaines aux congrès de la FDIF exaltent «une communauté d'intérêts » fondée sur la maternité. Si le registre maternant est une caractéristique classique des mouvements pacifistes, plusieurs des contributions de ce numéro soulignent la prégnance de l'expérience et de la rhétorique de la maternité dans les mobilisations

42 J. Siméant, « Protester/mobiliser/ne pas consentir. Sur quelques avatars de la sociologie des mobilisations appliquée au continent africain », Revue internationale de politique comparée, vol. 20, $\mathrm{n}^{\circ} 2,2013$, p. 125143. 
féminines ou mixtes qu'elles étudient. On observe ainsi à plusieurs reprises que la maternité est utilisée par les militantes et militants en tant que dispositif de sensibilisation à leur cause ou identité stratégique. C'est ce que montre par exemple Pierre-Jean Le Foll-Luciani à propos des communistes algériens qui privilégient aux mots d'ordre marxistes ou anticolonialistes des questions sociales «maternelles» (prix des denrées alimentaires, scolarisation, santé) pensées comme plus mobilisatrices pour le public féminin qu'ils entendent recruter. La mise en scène de l'identité maternelle sert ensuite aux protestataires à légitimer leurs actions militantes, qui transgressent par ailleurs les assignations de genre qui pèsent sur elles dans leurs autres sphères de vie (conjugale, familiale, professionnelle ou confessionnelle). C'est par exemple en jouant sur leur identité de mère ou d'épouse que des femmes de l'Union démocratique des femmes camerounaises protestent et pétitionnent pour la libération de leurs parents ou époux, emprisonnés pour leur engagement dans l'Union des populations du Cameroun (UPC) (R. Ndengue) ${ }^{43}$. C'est en jouant sur cette même identité féminine ou maternelle supposée étrangère à toute activité politique que des militantes communistes couvrent leurs actions de propagande, font passer des armes, et se protègent de la répression de l'administration coloniale (P.-J. Le Foll-Luciani).

Ce dernier exemple invite à penser la dimension genrée des modes d'action et des organisations ou collectifs militants, interrogeant par-là même la division sexuelle du travail militant. À l'image d'autres travaux sur le sexe du militantisme, les auteurs de ce numéro documentent le rôle de «leader-pont » (bridge leaders $)^{44}$ joué par les femmes, et l'importance des capitaux et réseaux proprement féminins dans l'appui aux organisations. Les militantes algériennes, communistes comme messalistes, se voient par exemple confier la responsabilité d'entretenir les réseaux de sociabilité militante, ou servent de «petites mains » à l'organisation des réunions (P.-J. Le Foll-Luciani, N. Sidi Moussa). C'est également les réseaux et lieux de sociabilité féminine (marchés, hammams, fêtes religieuses ou familiales) qui sont mobilisés pour enrôler de nouvelles recrues, ou servir de relais à l'action protestataire. Ophélie Rillon montre que la rue ou le marché, quotidiennement investis par des commerçantes, sont des lieux d'activité politique intense, questionnant

43 À l'instar de leurs homologues kenyanes étudiées par A. Tibbetts, « Mamas Fighting for Freedom in Kenya », Africa Today, vol. 41, 1994, p. 27-48.

44 B. Robnett, « African-American Women in the Civil Rights Movement, 1954-1965: Gender, Leadership, and Micromobilization », American Journal of Sociology, vol. 101, n 6, 1996, p. 1661-1693. 
ainsi la conception d'une partition stricte entre espace public et espace privé, entre activités domestiques, professionnelles et militantes. Les femmes jouent ensuite un rôle de soutien moral, logistique, financier au sein des diverses organisations étudiées, dont les hommes conservent le leadership. Et ce n'est pas sans ironie que des sœurs, filles ou épouses de militants du Parti communiste algérien (PCA) se trouvent confrontées au «moralisme sexuel et racial », pour reprendre les termes de Pierre-Jean Le Foll-Luciani, de leur frère, père ou époux militants pourtant empreints d'idées autrement progressistes. Les contradictions entre les mots d'ordre officiels d'émancipation de la femme et le fonctionnement concret des organisations sont souvent patentes. Elles montrent combien l'émancipation féminine officiellement défendue par le PCA, l'Union des femmes d'Algérie ou l'UPC reste une émancipation « sous contrôle ». Nedjib Sidi Moussa signale par exemple la centralité de la maternité patriotique dans le programme messaliste où les Algériennes sont pensées par les dirigeants du Mouvement national algérien avant tout comme des «gardiennes de la tradition ». Ce modèle d'émancipation est du reste formulé dans un espace idéologique concurrentiel où s'affrontent déjà les modèles promus respectivement par l'administration coloniale et le FLN. Il résulte de ces contradictions internes aux organisations militantes des conflits ouverts (P.-J. Le FollLuciani, R. Ndengue, N. Sidi Moussa), des jeux d'instrumentalisation réciproque (M. Idriss, R. Ndengue) ou encore des stratégies de résistance et de contournement lorsque les femmes recourent à des espaces ou organisations non mixtes (P. Barthélémy), s'emploient à tenir la façade de la «bonne » épouse ou camarade (R. Ndengue, C. Walker-Said), ou donnent des gages de moralité et nient a posteriori toute implication dans des actions jugées subversives (M. Idriss).

Il est une question qui illustre particulièrement les tensions ouvertes par les logiques patriarcales du militantisme : le recours des femmes à l'action violente (symbolique ou physique, et dans ce dernier cas, armée ou non). À rebours des représentations stéréotypées genrées - partagées notamment par l'administration coloniale - qui conçoivent la violence physique comme un attribut uniquement masculin, plusieurs des contributions rappellent à juste titre que les femmes peuvent aussi, de manière autonome et raisonnée, participer à des modes d'actions violents, parmi lesquels les émeutes de rue, les saccages de lieux publics, les bastonnades ou la lutte armée. Les auteurs de ce dossier ne se contentent toutefois pas de dénombrer des femmes prenant part à des événements violents. Ils cherchent plutôt à 
souligner ce que ces actions féminines violentes peuvent nous dire des logiques genrées du militantisme, et des rapports de genre plus généralement observés dans les sociétés étudiées. Tel est le cas des femmes mobilisées pour la départementalisation de Mayotte (M. Idriss) qui se désignent comme des soldates (sorodats) mais qui ont été qualifiées de «chatouilleuses » par des journalistes métropolitains et des militants mahorais. Une terminologie pour le moins surprenante et qui fait étrangement écho à d'autres femmes, issues des milieux populaires, que l'on surnomma «tricoteuses » ou encore «furies de guillotines »sous la Révolution française ${ }^{45}$. La minimisation et l'invisibilisation de la violence féminine par les hommes ont servi à réaffirmer un ordonnancement « traditionnel » des rôles féminins et masculins, où les militantes ont été exclues du champ de la politique conventionnelle, contre la subversion que représentaient les actes de « chatouilles » dévirilisant les hommes politiques visés. Ophélie Rillon montre à l'inverse que, dans le contexte de compétition exacerbée entre les partis politiques au Soudan français, les mobilisations et batailles de rue sont confiées aux militantes de base issues des classes populaires. Les violences féminines ne sont alors pas envisagées comme une subversion des rôles de genre mais bien comme le produit du façonnement genré des organisations partisanes et le fruit d'une reproduction des hiérarchies sociales de genre et de classe. Dans le champ militant, l'action violente est alors considérée comme « un travail de femmes ${ }^{46}$, et précisément de femmes du peuple, tandis que les femmes éduquées participent aux réunions politiques. Si les auteurs de ce dossier ne se réfèrent pas explicitement aux travaux sur l'intersectionnalité, il est clair que cette approche irrigue leur réflexion, et qu'ils ne souscrivent pas à une vision réifiée ou homogénéisante de la « catégorie femme », ou de l'un ou l'autre des deux sexes. Leur étude des rapports de genre reste attentive à l'imbrication de ces derniers dans d'autres rapports de domination : classe sociale, «ethnie » ou « race», mais aussi âge - ce dernier trop souvent ignoré sur les terrains occidentaux est pourtant fondamental en Afrique ${ }^{47}$.

45 D. Godineau, Citoyennes tricoteuses. Les femmes du peuple à Paris pendant la Révolution française, Paris, Perrin, 2004.

46 On retrouve ce schéma en Tanzanie au sein de l'Union nationale africaine du Tanganyika étudié par S. Geiger, « Tanganyikan Nationalism as 'Women's Work' : Life Histories, Collective Biography and Changing Historiography », The Journal of African History, vol. 37, n 3, 1996, p. 465-478.

47 Sur les relations entre «aînés » et «cadets » comme rapport social de domination, voir G. Balandier, Anthropo-logiques, Paris, Presses universitaires de France, 1974. 
La question de la violence interroge en définitive la fabrique des rapports de genre au cœur des luttes sociopolitiques dans cette période singulière de la fin de l'Empire. Quelles ont été les conséquences des luttes auxquelles prirent part certaines femmes ? Les rapports de pouvoir entre les sexes ont-ils été redéfinis ou reproduits au cours de cette période ? La réponse n'est pas définitive. On observe tout d'abord que l'engagement militant a pu être émancipateur à bien des égards pour plusieurs militantes. Pascale Barthélémy montre combien l'accès aux réseaux féminins transnationaux et les voyages à l'étranger sont importants dans les carrières militantes alors en construction dans la mesure où ils offrent de nouvelles ressources (oratoires, idéelles et matérielles), ouvrent de nouveaux horizons, permettent de négocier une autre répartition des rôles au sein du couple. Pierre-Jean Le Foll-Luciani, quant à lui, révèle les conséquences biographiques singulièrement émancipatrices des expériences militantes «ordinaires » mais surtout «extrêmes » (lutte armée, clandestinité, exil, répression et tortures). Des militantes algériennes témoignent ainsi avoir gagné en estime d'elles-mêmes, conquis une liberté (notamment de mouvement) et adopté une nouvelle hexis (port du pantalon et des cheveux courts, dévoilement). Le bouleversement des divisions de race, de sexe et de confession est également frappant dans les couples mixtes communistes ou messalistes. Une des rares organisations comme le PCA à réunir dans les mêmes cellules des femmes et des hommes européens, algériens, musulmans ou juifs, illustre combien l'expérience militante, celle de la clandestinité et de la répression, peut transformer les rapports de genre et de race. Pour finir sur ce point, il faut noter que les contributions de ce dossier invitent à explorer davantage encore les arrangements familiaux et conjugaux, qui rendent possible ou non le militantisme, ainsi que l'articulation des sphères de vie dans le cours des mobilisations et carrières militantes. S'agissant de l'étude de la fabrique des rapports de genre à l'intérieur des luttes, on aurait tort de lire le féminisme différentialiste défendu par les dirigeants messalistes ou les jeunes militantes et militants camerounais des associations chrétiennes à l'aune des courants féministes plus radicaux qui prirent forme quelques décennies plus tard, et donc de disqualifier le féminisme différentialiste en soulignant uniquement son « conservatisme » ou son caractère « rétrograde». Quelle que puisse être la validité des étiquetages ou des taxinomies militantes («tradition» contre «modernité », «conservatisme » contre «féminisme »), l'engagement de chrétiennes camerounaises dans le militantisme social et la défense des valeurs familiales et conjugales a aussi ouvert la voie à des réformes et à des 
trajectoires d'émancipation. Rose Ndengue montre par exemple que l'Association des femmes camerounaises qui s'inscrivait dans le cadre de la «mission civilisatrice » prônée par l'administration coloniale en développant des programmes d'actions sociales visant à améliorer la condition des femmes pauvres, réclamait aussi l'égalité des droits en matière de vote ; ces deux revendications étaient alors pensées de manière complémentaire, la première légitimant la seconde. De son côté, Charlotte Walker-Said montre que le militantisme religieux, en dépit de l'émancipation féminine contrôlée qu'il promeut, a aussi rendu possibles des formes d'émancipation féminine par l'accès au travail ou aux soins médicaux. Les aspirations de réforme sociale des jeunes croyantes camerounaises illustrent que le militantisme religieux et social s'est offert comme mode alternatif d'action féminine permettant d'imaginer un avenir personnel et différents modèles de construction nationale dans le contexte incertain des années 1950.

A posteriori, l'époque coloniale tardive apparaît comme une parenthèse «enchantée » où des femmes et des hommes ont fait bouger les frontières des identités ou des rôles féminins et masculins sans que leurs actions aient été relayées et véritablement mises en œuvre par les États postcoloniaux. L'étude de la période d'incertitude et de bouleversements des dernières décennies de la colonisation montre toutefois que les issues politiques comme sociales étaient ouvertes. 